\title{
Antioxidant Activities of Resveratrol Loaded Poloxamer 407: An In Vitro and In Vivo Study
}

\author{
${ }^{1}$ Ali A. Al-Jubori*, ${ }^{1}$ Ghassan M. Sulaiman, ${ }^{2}$ Amer T. Tawfeeq \\ ${ }^{1}$ Division of Biotechnology, Department of Applied Sciences, University of Technology, Iraq \\ ${ }^{2}$ Department of Molecular Biology, Iraqi Center for Cancer and Medical Genetics Research, Mustansiriyah \\ University, Iraq
}

\section{Article information}

\section{Article history:}

Received: June, 10, 2021

Accepted: July, 26, 2021

Available online: September, 18, 2021

Keywords:

Resveratrol,

Poloxamer 407,

DPPH,

In vivo,

Animal model

\section{*Corresponding Author:}

Ali A. Al-jubori

ali.adnan.aljubori@gmail.com

\begin{abstract}
Resveratrol (RES) is a bioactive molecule with potent antioxidant properties, and it constitutes an essential component of herbal medicine. This study was designed to use a nano-syntheses approach to encapsulate RES in Poloxamer 407 nanoparticles. This type of nanoconstruction has been employed in a variety of biological applications, both in vitro and in vivo. The contribution of this nano-construct is to increase antioxidant activity over the pure RES was investigate. 1,1diphenyl-2-picryl hydrazyl (DPPH), and hydrogen peroxide radical scavenging assays were utilized in vitro. RES-loaded poloxamer 407 showed concentration-dependent scavenging action in the range of 20$80 \mu \mathrm{g} \cdot \mathrm{ml}^{-1}$, with a maximum activity of $80.1 \%$ at $80 \mu \mathrm{g} \cdot \mathrm{ml}^{-1}$. Whereas pure RES had a decrement of $61.7 \%$. While the activity of positive control (Vit. C) was 93.2\%. The magnitude of blood hemolysis examination was $3.9 \%$ at $80 \mu \mathrm{g} \cdot \mathrm{ml}^{-1}$. That may indicate RES-loaded poloxamer 407 provide significant red blood cell protection. The pure RES did not prevent the appearance of abnormal cells (echinocytes), and that was cured by RES-loaded poloxamer 407 . Finally, the safety of RES-loaded poloxamer 407 was assessed in vivo. Male mice were invested to detect the functions of their liver and kidney. A histopathological study was included as well. The findings showed that RES-loaded poloxamer 407 might have superior characters as a drug delivery system, nutritional supplements, and may be used in pharmaceutical products.
\end{abstract}

DOI: 10.53293/jasn.2021.3809.1046, Department of Applied Science, University of Technology

This is an open access article under the CC BY 4.0 License

\section{Introduction}

According to the International Agency for Research on Cancer's (IARC) most recent study, cancer causes elevated to 8.8 million per year, with the rate expected to rise to 13.2 million by 2030 [1]. Traditional medicine based on bioactive natural compounds has been used for ages in many cultures worldwide [2]. Naturally occurring phytochemicals have gotten a substantial puss because of their wide spectrum of therapeutic actions. These bioactive natural chemicals are found in a variety of edible plants and have 
good medicinal efficacy. They are integrated into the plants' system to defend them against viruses, parasites, and other diseases. In recent years the bioactivity of curcumin, genistein, berberine, resveratrol, quercetin, and boswellic acid has been investigated extensively. Depending on their chemical structure, dietary phytochemical products are classified into alkaloids, polyphenols, and nitrogen compounds [3]. Polyphenols include numerous bioactive compounds such as phenolic acids, flavonoids, stilbenes, coumarins, and lignans. Stilbenes, particularly trans-resveratrol, have got the attention as a result of their wide distribution in plants and extensive bioactivity. Resveratrol (RES) was found in a variety of vegetation, including peanuts, blueberries, legumes, rhubarb, grapes, eucalyptus, and other grasses. Antiinflammatory, antioxidant, and hepatoprotective properties activities were associated with RES [4-6]. Besides that, other pharmacological features were correlated like cardioprotection, disaggregation of the platelet, and vaso-relaxant properties. It also suppresses the virus of human immunodeficiency and herpes simplex [7]. Its anti-angiogenic property was also suggested, and it was recommended to be used in cancer prevention $[8,9]$. This compound character as an anti-cancer factor against a variety of malignancies was described as early as the nineteenth by Jang et al [10].

Soleas et al. compared the anti-carcinogenic properties of four polyphenols. Catechin, quercetin, gallic acid, and trans-resveratrol were administrated topically over skin tumor lesions twice a week for 18 weeks. The mice previously have a potent carcinogen topically in the same skin area. Results indicated that transresveratrol was the second to inhibit tumor formation and first to be absorbed by mice skin [11, 12]. The Resveratrol anticancer effect displayed a dual capability; it protects normal cells from impairment and suppresses malignant cell proliferation. It proved to play a crucial role in tumor cell apoptotic signaling via direct stimulating of caspases cascade and inhibiting anti-apoptotic pathways. All that due to the increment of p21 level and hence p53 activity. Because of this kinship, p53 may definitively stop cell proliferation [13, 14]. Furthermore, RES anticancer efficacy has been observed to be improved when administered in conjunction with other chemotherapy medicines $[15,16]$. Studies that shown RES antiproliferative properties in animal models was faced by limitation of its low water solubility, chemical instability, as well as inadequate absorption across biological membranes to allow its use for human. Whoever, its quick metabolization hinders the research in its anticancer potency, its effectiveness in cancer prevention and treatment [17]. To circumvent these constraints, new nano-formulations can be developed to approach it is potentials. Resveratrol nanoparticle-based formulations have been developed to increase its absorption, and to deliver the right quantity of it to the tumor tissue. Polymeric nanoparticles, liposomes, and other nano-formulations for RES delivery are among the new nanoformulations. These systems improve the solubility, stability, and permeability of water across biological membranes, as well as the increased permeation and retention effect (EPR) at tumor locations [18]. Thus, the present study was designed to evaluate the anti-oxidative stress activity of pure RES and formulated RES-loaded poloxamer 407. In addition, the animal model was employed to elucidate the toxicity issue against the liver and kidneys of mice. 


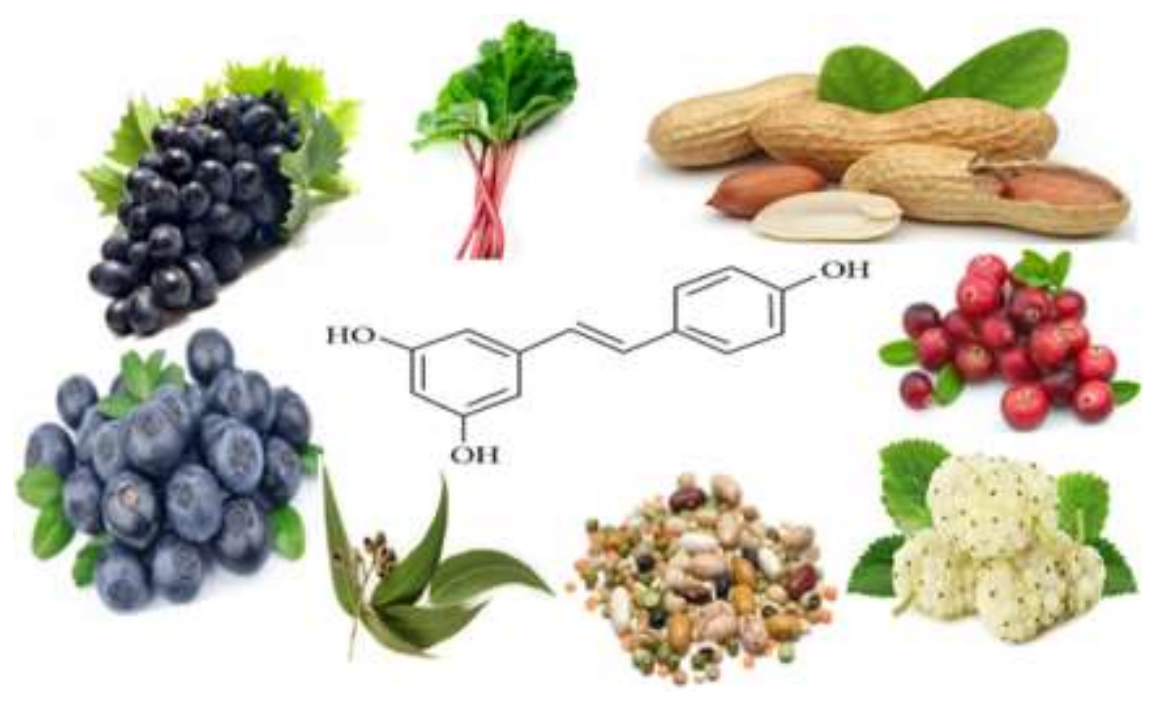

Figure 1: Chemical structure and rich sources of RES [4-6].

\section{Material and Methods}

\subsection{Chemicals and Reagents}

Ethanol (Chem-lab,Belgium), Resveratol (RES; 99\% purity), Poloxamer 407 (P407), ascorbic acid (Vitamin C) and 1-diphenyl-2-picrylhydrazyl (DPPH), All of these ingredient purchased from Sigma Chemical Co. USA, standard fish DNA (BDH, England).

\subsection{Fabrication of RES-Poloxamer 407 Nanoparticles}

RES-Poloxamer 407 was prepared by participation method as previously reported by Sulaiman et al [19]. With minor modifications, briefly, RES $(100 \mathrm{mg})$ was dissolved in $5 \mathrm{~mL}$ of ethanol. The resulting solution was added dropwise onto $40 \mathrm{~mL}$ of Poloxamer-407 solution $(500 \mathrm{mg}$ with $100 \mathrm{~mL}$ of deionized distilled water).

After that, the mixture was maintained under continuous stirring at $100 \mathrm{rpm}$ for $24 \mathrm{~h}$ to evaporate the ethanol. The resultant RES-loaded poloxamer 407 was subjected to homogenization by vortex, sonication for 12 min and water bath $40^{\circ} \mathrm{C}$. After that, the system was left for equilibrium for $24 \mathrm{~h}, 25^{\circ} \mathrm{C}$ as seen in figure (2).

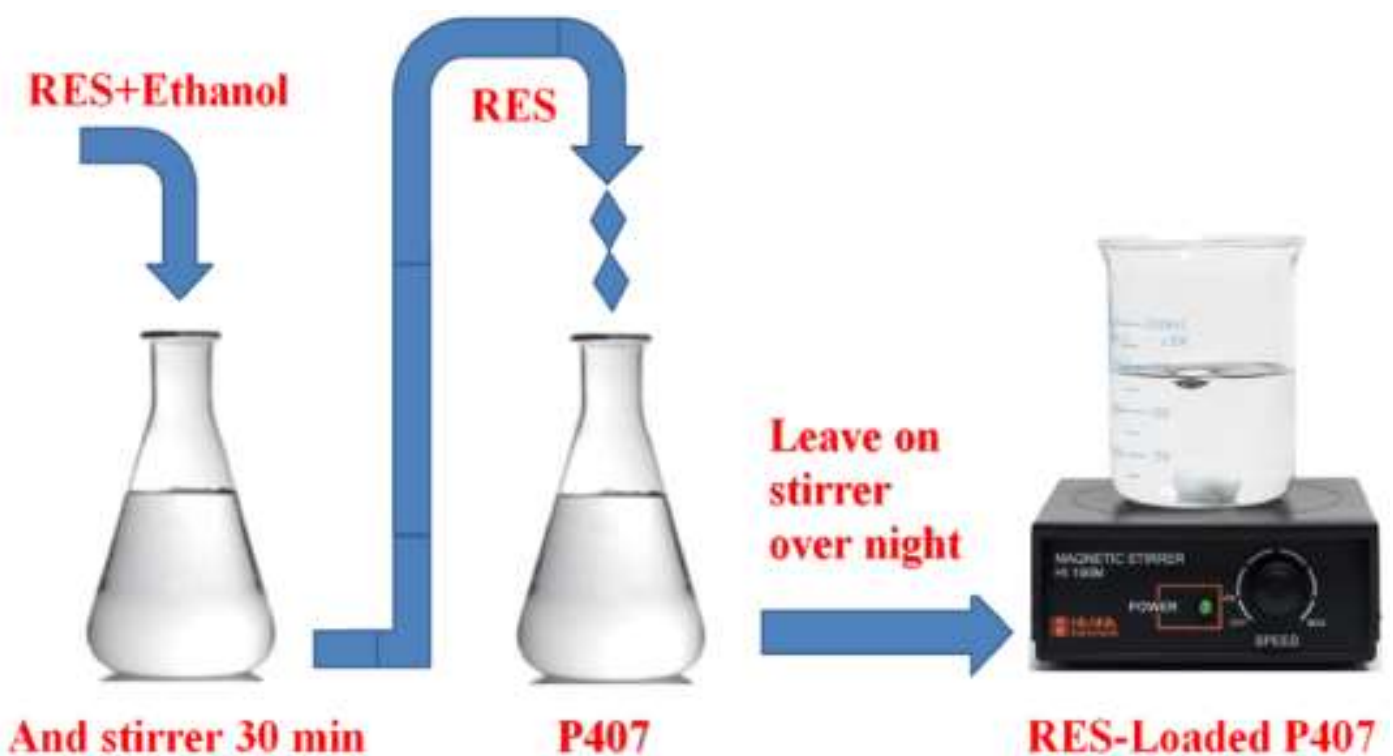

Figure 2: Preparation of RES-loaded poloxamer 407 nanoparticles. P407: poloxamer407, RES: resveratrol. 


\subsection{Antioxidant Activity}

DPPH (1, 1-diphenyl-2picrylhydrazyl) radical scavenging activity of pure-RES and RES-loaded poloxamer 407 was valuated as followed according to Hao, et al [20]. With some modification, typically, absolute ethanol 500 $\mu \mathrm{L}$ was add to different tubes and mixed with $0.5 \mathrm{~mL}$ different concentrations of pure RES and RES-loaded poloxamer 407. (20, 40 and $\left.80 \mu \mathrm{g} \mathrm{mL}^{-1}\right)$ and then $500 \mu \mathrm{L}$ of (DPPH) $(60 \mu \mathrm{M})$ was added into all tubes and brooded about 30 min at room temperature. The negative control was DPPH with ethanol only and the positive control was $5 \mu \mathrm{g} \mathrm{mL}$ ${ }^{1}$ of vitamin C. After that the absorbance of all samples was tested at $517 \mathrm{~nm}$ utilizing UV-VIS spectrophotometry (Metertech, Germany). The percent of DPPH scavenging efficacy was measured utilizing the next equation:

$$
\text { Anti - oxidant capacity }(\%)=\frac{A c-A s}{A c} \times 100
$$

Ac represents the peak intensity of DPPH and as is the peak of pure RES or RES-loaded poloxamer 407 sample solvent.

\subsection{DNA Damage Assay}

Quantification of antioxidant capacity was determined spectrophotometrically by using DNA damage assay. In this assay, the absorbance ratio of DNA sample about (A260/A280) which were treated with varied quantities (20, 40, and $80 \mathrm{~g} \mathrm{~mL}^{-1}$ ), equal volumes of pure RES and RES-loaded poloxamer 407 and standard DNA solution were mixed with a mixture of $1 \times 10^{-5} \mathrm{M}\left(\mathrm{H}_{2} \mathrm{O}_{2}\right)$ hydrogen peroxide solution and incubated for around 10 minutes at $37^{\circ} \mathrm{C}$ temperature, after this period the effect was determined by evaluated the wavelength of the solution at $260 \mathrm{~nm}$ utilizing Ultraviolet-Visible spectrophotometer and in the current study $5 \mathrm{mg} \mathrm{mL}^{-1}$ of Vitamin C Ascorbic acid was used as positive control [21].

\subsection{Human Blood Collecting}

Human blood samples were freshly collected from five healthy volunteers and divided into tubes containing the anticoagulant agent (heparin), according to the National Institute of Health and Food and Drug Administration and Helsinki's declaration and regulation as a statement of ethical principles.

\subsubsection{Blood Compatibility Assay}

Hemolytic toxicity of pure-RES and RES-loaded poloxamer 407 was carried out according to Sulaiman, et $a l$ [22]. With some modification, briefly, $200 \mu \mathrm{L}$ of the separated blood was diluted with $1600 \mu \mathrm{L}$ normal saline. Along with the controls, $200 \mu \mathrm{L}$ of varying concentrations of pure RES and RES-loaded poloxamer 407 (20, 40, and 80 $\left.\mu \mathrm{gmL}^{-1}\right)$ were added to this diluted blood. As a positive control (100 percent lysis), pure water was used, while normal saline was used as a negative control ( 0 percent lysis). The samples were then centrifuged for 5 minutes at $100 \mathrm{rpm}$ after being incubated at $37^{\circ} \mathrm{C}$ for $1 \mathrm{hr}$ in a water bath. A UV-Vis spectrophotometer was used to measure the absorbance at $540 \mathrm{~nm}$, and the percentage of hemolysis was calculated using the method below.

$$
\text { Hemolysis } \%=\left(\frac{\text { Abs }- \text { Abc }(- \text { ve })}{\text { Abc }(+ \text { ve })-\mathbf{A b}(-\mathbf{v e})}\right) \times 100
$$

Abs is absorbance of standard and Abc is absorbance of control.

After that, one drop of blood from each sample was used to produce a smear on the slide, which was then allowed to dry at room temperature. Slides were stained for 10 minutes with 5 drops of Leishman stain and then rinsed with distilled water. The cells examined under a light microscope at magnification of 40 (H.P.F). Other slides were examined under fluorescent microscope.

\subsection{Histopathological Changes}

Swiss albino male mice weighing 22 - $25 \mathrm{~g}$ and aged 4 to 5 weeks were used. Mice were kindly provided by Iraqi Center for Cancer and Medical Genetic Researches, University of Al-Mustansiriyah, Baghdad, Iraq. The mice were housed in a 12:12 hr light/dark cycle with a constant supply of water and food at a temperature of $24^{\circ} \mathrm{C}$. and a humidity of $55 \%$. All procedures were approved by the Animal Care and Ethics Committee at Biotechnology Division, Applied Sciences Department, University of Technology, Baghdad, Iraq. 
Nine mice were randomly assigned to one of three groups (three animals per group): Group I got no treatment and were given normal saline as an untreated control, Group II got a low dosage (20 $\left.\mathrm{mg} \mathrm{Kg-}^{1}\right)$ of RES-loaded poloxamer 407, and Group III got a high dosage $\left(80 \mathrm{mg} \mathrm{Kg-}^{1}\right)$ of RES-loaded poloxamer 407. Mice were injected intraperitonealy (IP) with $100 \mu \mathrm{L}$ of RES-loaded poloxamer 407 solution containing 20 and $80 \mathrm{mg} \mathrm{Kg}^{-1}$ for three days a week (the final volume was varied according to the animal weight). Normal saline ( $0.1 \mathrm{~mL}$ mouse- ${ }^{1}$, As a negative control) was injected three times a week into the control group [23].

During the experiment, animals' weight and behavior were meticulously documented. After 7 and 14 days of therapy, mice from each group were sacrificed. The liver and kidneys from the animals were then taken. In both non-treated and treated mice, the organs were washed in PBS and fixed in 10\% formalin before being embedded in paraffin for histological assessment. A microtome was used to create slices, which were then stained with hematoxylin and eosin $(\mathrm{H} \& \mathrm{E})$. The sections were processed and stained in accordance with a standard approach employed in histopathology laboratories [24].

\subsection{Statistical Analysis}

The submitted data was analyzed using the SPSS statistical software (Version/18.0; SPSS Inc., Chicago, IL). (ANOVA) was performed for analysis of variance to see whether there are any statistically significant differences between the means of the studies. A statistically significant p-value of 0.05 was considered.

\section{Results and Discussion}

\subsection{Fabrication of RES-Loaded Poloxamer 407 Nanoparticles}

Figure (3) represents the preparation of RES-loaded poloxamer 407. As seen in the figure, RES-loaded poloxamer 407were first formulated using dilution and increase the solubility in water through nano participation method .In these technique, an organic solution of the $\mathrm{P} 407$ polymer is emulsify in an aqueous solution with a surfactant. Then, removing the organic solvent by stirring and this process permits formation of nanoparticles. the poloxamer give good stability and homogenized for long time as see in figure (4).When trying to dissolve pure RES in water, we noticed that it is very difficult to dissolve, as the solution appears foggy, with the presence of clear insoluble particles in the solution. But after applying the precipitation technique and converting pure RES to RES-loaded poloxamer 407 by changing the color of the mixture to gray, we found that the developed RES became homogeneous and completely soluble. The increased water solubility of modified RES may be due to their higher surface area, which favors dissolution. The solubility of a medicine is frequently inherently connected to the particle size of the medication; as a particle grows smaller, the surface area to volume ratio increases. The increased surface area allows for better contact with the solvent, resulting in increased solubility [25]. Previous studies have discovered a hyperbolic relationship between particle size and surface specific dissolving rate adjusted for solubility. Similar outcomes have been seen when the particle size of active substances is reduced to nanoparticle size, resulting in improved solubility and bioavailability [26].

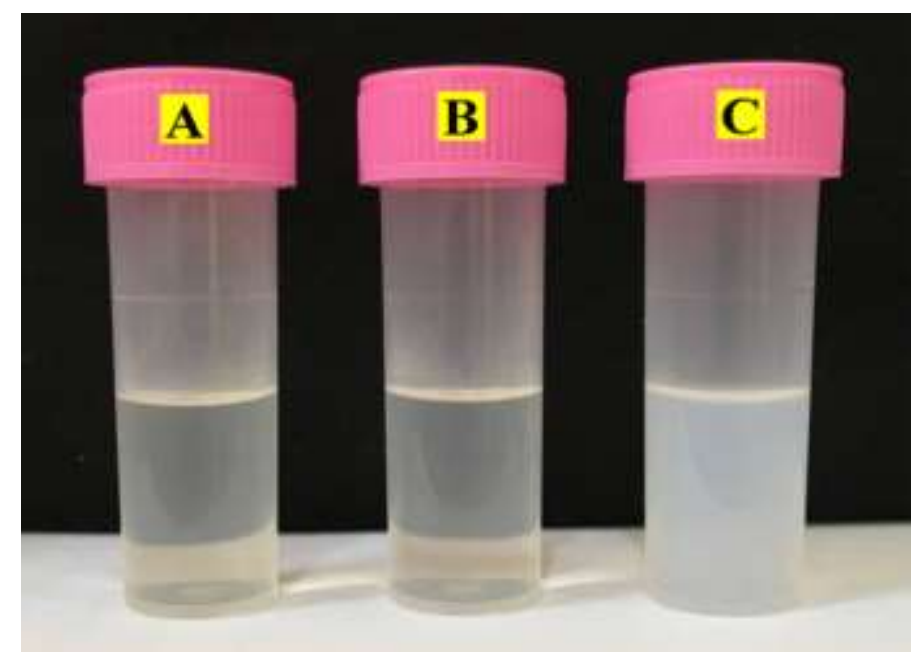

Figure 3: Stages of the chemical reaction for P407 loaded with RES, the color change is detected with reaction progrition. A: RES, B: P407, C: RES-loaded P407. 


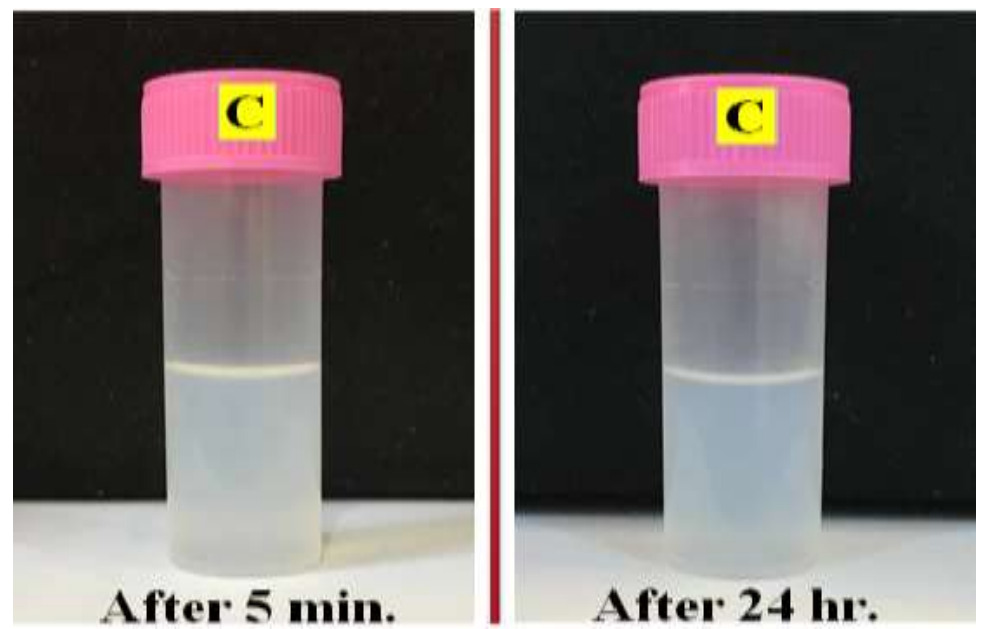

Figure 4: The high stability of the RES-loaded poloxamer 407 after $24 \mathrm{hr}$ of preparation. [(C) RES-loaded poloxamer 407 .

\subsection{Antioxidant Capacity of (DPPH Radical Scavenging Assay)}

The radical scavenging mechanism with DPPH (1,1-diphenyl-2-picrylhydrazyl) is based on electron transfer to the compound and generates a rich purple hue in ethanol solution [27]. These free radicals, which are stable at $25^{\circ} \mathrm{C}$, turn colorless to light yellow when they combine with an antioxidant molecule as a hydrogen donor. The reduction level in the reaction is proportional to the absorbance quantity of the antioxidants capability. Figure (5) describes the antioxidant activity of natural RES and RES-loaded poloxamer 407 using three different concentrations. The results revealed that the RES in nano-structure had a greater scavenging capacity than the normal RES, and depended on the concentration used. The results demonstrate that RES in its natural structure reduces the level of free radicals of DPPH at $80 \mu \mathrm{g} \mathrm{mL}^{-1}$ by $61.67 \%$ and it was superior to other concentrations used. While the RES in its nano-structure achieved $80.1 \%$ at the same concentration. The positive control generated $93.22 \%$ of antioxidant activity for DPPH inhibition. This higher activity of RES nano-structure in scavenging the radicals of DPPH than that of the normal structure of RES can be explained by enhanced dissolvability. It was observed that phenols and polyphenols, in general, have a higher DPPH scavenging influence if they were in the form of nanostructures rather than their normal form. This was also seen in RES, hesperidin, and quercetin. The RES nanoparticles scavenging ability toward DPPH showed a significant superiority compared to its natural form at the same concentration. RES, as a hydrophobic polyphenol, has already been confirmed to possess relatively strong antioxidant activity due to its hydrogen donating strength $[28,29]$. The elevation of the biological efficiency of flavonoids when turned to nanoparticles was observed. This elevation comes as a result of increment in their specific surface area, surface energy, and catalytic reactivity [30]. This can explain why RES-loaded poloxamer407 showed the highest DPPH scavenging ability. The results suggested that turning RES to nanoparticle form can remarkably enhance its antioxidant potential. 


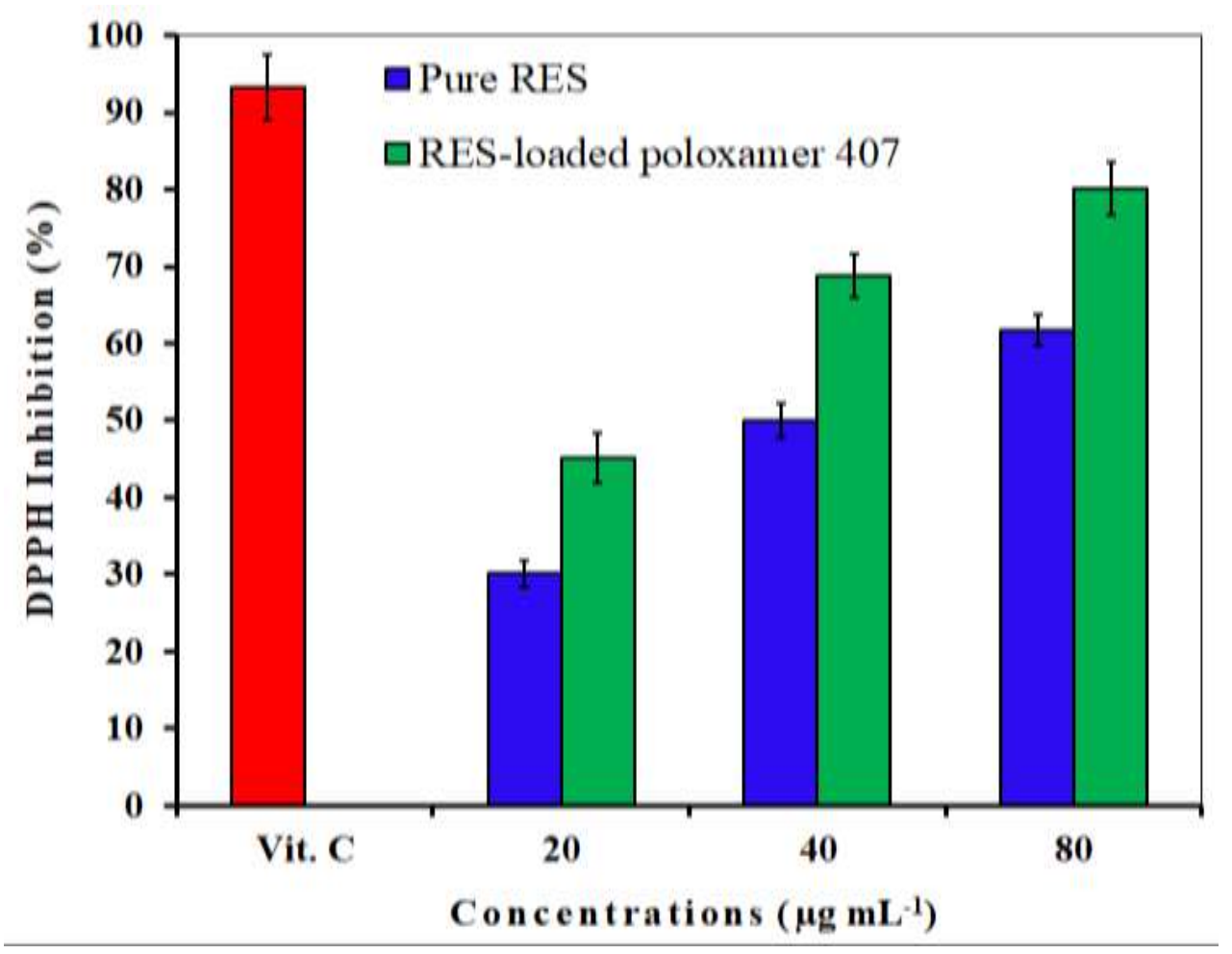

Figure 5: Free radical scavenging activity of pure RES (Blue color) and RES-loaded poloxamer 407 (Green color) using DPPH at three different concentrations.

\subsection{DNA Damage Assay}

One of the acceptable models to measure the bio-protective influence of the natural structure of polyphenols compared to its nano-structure form is it's sparing of DNA damage induced by hydrogen peroxide $\left(\mathrm{H}_{2} \mathrm{O}_{2}\right)$. Hydrogen peroxide is a non-radical compound, but it is a highly reactive molecule that's generated in large amounts in living cells [31]. $\mathrm{H}_{2} \mathrm{O}_{2}$ is produced by $\mathrm{O} 2 \bullet-$ dismutation and other enzyme-mediated processes, and it takes part in the oxidation reaction via a non-radical pathway [32]. When the superoxide $(\mathrm{O} 2 \bullet-)$ radical reacts with $\mathrm{H}_{2} \mathrm{O}_{2}$, extremely reactive hydroxyl radicals are formed, which can be a source of additional damaging species, such as hydroxyl radicals $(\mathrm{OH})$. This will attack the guanine base in the double helix and cause DNA damage. As indicated in table $1, \mathrm{H}_{2} \mathrm{O}_{2}$-induced DNA damage was reduced when the normal structure of RES or RES-loaded poloxamer 407 was added to the reaction at three quantities $\left(20,40\right.$, and $\left.80 \mu \mathrm{g} \mathrm{mL}^{-1}\right)$. The RES at its nano-structure at $80 \mu \mathrm{g}$ $\mathrm{mL}^{-1}$ being the most effective. The result indicated that the reaction tube becomes less absorbent when the natural RES or RES nano-structure concentration rises with advantage to RES nano-structure. This type of reaction can be connected to RES antioxidant properties as a natural phenolic molecule, generally, these molecules are considered potent antioxidants. The intensified level of antioxidant activity of the modified RES as demonstrated through this result resembles the beneficially of turning RES to nano-structure. Introducing this artificial structure to practical application, illnesses caused by free radicals, such as cancer, probably can be treated while causing no damage to normal cells [33].

In this sense, turning RES into a more soluble compound such as nano-structure as in this case may preserve DNA by lowering oxidative DNA damage caused by oxidative stress resulting from the presence of $\mathrm{H} 2 \mathrm{O} 2$ and improving DNA repair by modulating DNA repair enzyme production. This confirms that when cells are subjected to high levels of oxidative stress, diets high in RES are more efficient in boosting DNA repair. Lowering the intracellular oxidation level can help protect cells and tissues from neurodegeneration, cancer, diabetes, and obesity-related illnesses[34]. 
Table 1: The influence of natural RES and RES-loaded poloxamer 407 on the DNA molecule and its preservation from the effect of oxidation reaction.

\begin{tabular}{|c|c|c|c|c|}
\hline Compounds & $\begin{array}{l}\text { Concentration } \\
\left(\mu \mathrm{g} \mathrm{mL}^{-1}\right)\end{array}$ & DNA & $\begin{array}{c}\mathrm{H}_{2} \mathrm{O}_{2}+ \\
\text { DNA }\left(\mathbf{1} \times 10^{-5}\right) \\
\text { M }\end{array}$ & $\begin{array}{c}\mathrm{H}_{2} \mathrm{O}_{2}+ \\
\text { DNA }\left(1 \times 10^{-5}\right) \mathrm{MI} \\
+ \text { Compound }\end{array}$ \\
\hline \multirow[t]{4}{*}{ natural RES } & 0.0 & $0.248 \pm 0.021$ & 0.355 & $0.355 \pm 0.01$ \\
\hline & 20 & & & $0.311 \pm 0.02$ \\
\hline & 40 & & & $0.278 \pm 0.02$ \\
\hline & 80 & & & $0.237 \pm 0.02$ \\
\hline \multirow{4}{*}{$\begin{array}{c}\text { RES-loaded } \\
\text { poloxamer } 407\end{array}$} & 0.0 & $0.248 \pm 0.021$ & 0.355 & $0.355 \pm 0.01$ \\
\hline & 20 & & & $0.283 \pm 0.02$ \\
\hline & 40 & & & $0.249 \pm 0.03$ \\
\hline & 80 & & & $0.221 \pm 0.01$ \\
\hline
\end{tabular}

\subsection{Blood Compatibility Assay}

Any parenteral administration technique must have low or minimal hemolytic activity. Therefore, the interactions between red blood cells and the prepared formulations were studied and both natural RES and RES of the nanostructure were included in the study. The hemolysis impact on RBCs was measured after exposing the blood to three different concentrations of natural RES and RES nano-structure $\left(20,40\right.$, and $\left.80 \mu \mathrm{g} \mathrm{mL}^{-1}\right)$, as well as normal saline and distilled water as negative and positive controls, respectively. The effects of RES-loaded poloxamer 407 were shown to be minor. The proportion of hemolysis of RES-loaded poloxamer 407 was below the permissible criterion $(1.8,2.6$, and $3.9 \%$, respectively) of less than 5\% hemolysis at concentrations of 20 to $80 \mu \mathrm{g}$ $\mathrm{mL}^{-1}$ respectively. RBCs were shown to be less affected by RES nano-structure than by normal RES. Because of the high polyunsaturated fatty acid composition of their membranes and their high cellular oxygen and hemoglobin concentrations, erythrocytes are especially vulnerable to oxidative damage [35]. Under this in vitro setting, the results showed that RES-loaded poloxamer 407 protects human RBCs from hemolytic damage may be due to decreases oxidative stress $[36,37]$. This impact has been linked to a variety of processes, including membrane integrity maintenance, changes in cell membrane proteins and lipids, and changes in cell membrane water content. The potential of RES to integrate into cell membrane appears to be critical for the efficiency of polyphenol chemicals to maintain the integrity of the cells [38].

Lower concentration range, notably of the normal or natural RES, had more defective consequences on RBCs, causing cell membrane disintegration, and this impact was similar to that of the positive control. This could be due to the less solubility of RES making RBC susceptible to more water molecules. This was not observed with RES loaded poloxamer 407 Light microscopy images of the RBCs presented in figure (6); the images of hemolysis assay demonstrated no harmful action on the RBCs morphology. Because of the presence of more soluble RES and the major role that natural groups of polyphenols play in biocompatibility, RES-loaded P407 showed dramatically reduced hemolysis [39]. These findings demonstrated that RES-loaded poloxamer 407 was ready for systemic administration, with less than 5\% hemolysis. According to the American Society for Testing and Materials' E252408 standard [40]. Normal or natural RES caused red cells to suffer morphological changes from their typical discoid form to echinocytes as indicated in previous studies [41]. Foreign substances cause erythrocytes to shift form owing to the unequal expansion of the red cell membrane's two monolayers. When the chemical enters the inner monolayer, it produces stomatocytes, and when it enters the outer moiety, it produces spiculated-shaped echinocytes. The fact that natural RES stimulated the production of echinocytes suggests that it was introduced into the erythrocyte membrane's outer leaflet [42]. 

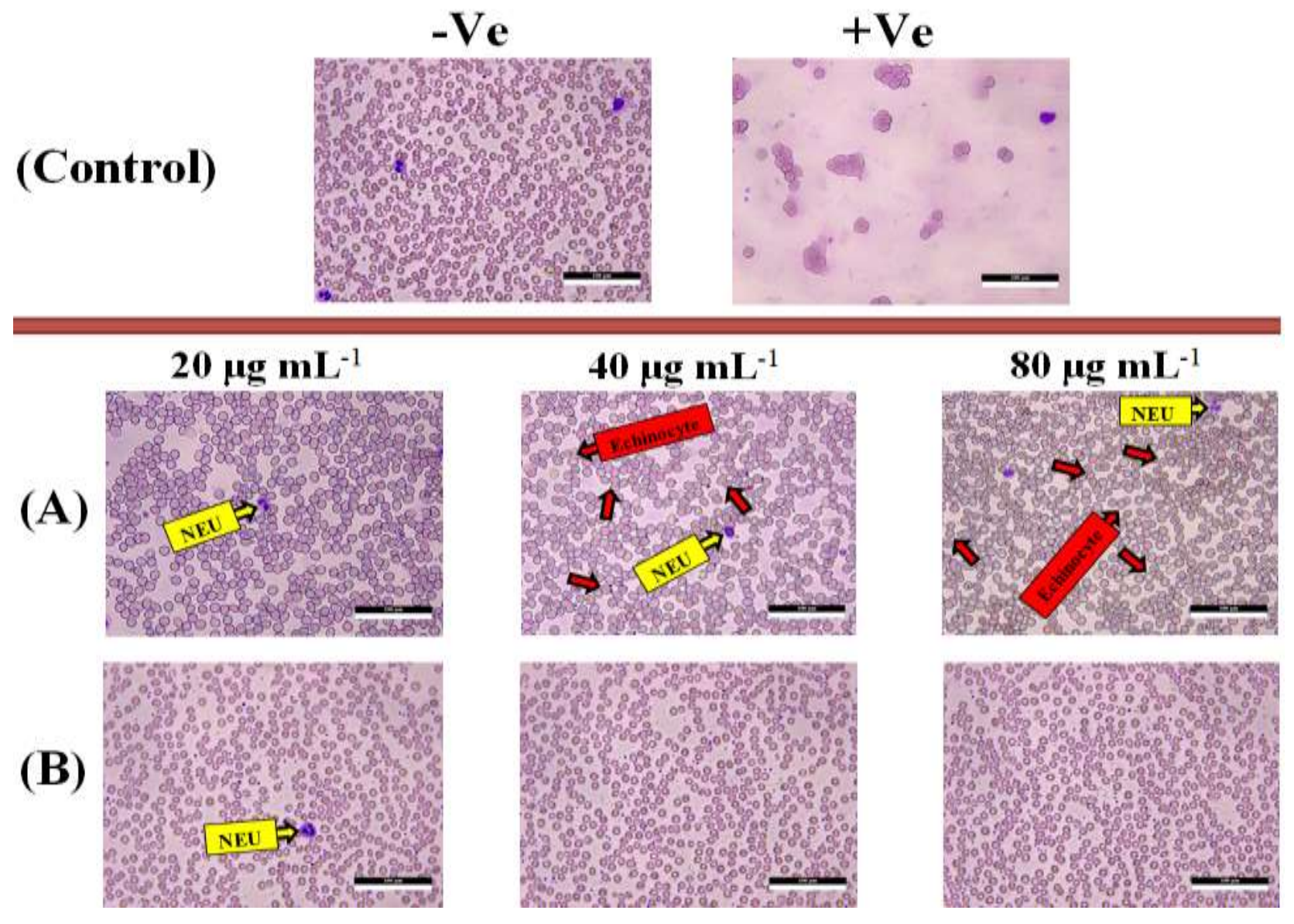

Figure 6: Light microscopic images showing the hemolytic toxicity: (A): Normal or natural RES (B): RES-loaded poloxamer 407 at concentrations $\left(20,40\right.$ and $\left.80 \mu \mathrm{g} \mathrm{mL}^{-1}\right)$ along with the positive and negative controls. * the yellow arrow is Neutrophil, while red arrow is the abnormal cells (Echinocytes) due to the effect of pure RES on their walls. Scale bar: $100 \mu \mathrm{m} .-\mathrm{Ve}$ and $+\mathrm{Ve}$ : negative and positive control, respectively.

\subsection{In Vivo Histopathological Changes}

In vivo experiments were conducted to assess the toxicity of RES-loaded poloxamer 407 using a mouse model. It can be noted that administration of 20 to $80 \mathrm{mg} \mathrm{Kg}^{-1}$ for 14 days did not result in death, and there were no statistically significant variations in body weight between the RES-loaded poloxamer 407 -treated animals and the control mice $(\mathrm{P} \geq 0.05)$. However, the body weight of mice before treatment were 20.0 \pm 0.40 and $21.5 \pm 0.82$, respectively, while, after treatment were $29.8 \pm 0.80$ and $30.2 \pm 0.64$, respectively. Furthermore, neither the control nor the treated groups showed any aberrant clinical indications or behavior. When taken as a whole, the RESloaded poloxamer 407 administration had no obvious impairment in mice, and they stayed healthy until the end of the trial. After the experiment, necropsy revealed no macroscopic organ alterations in both treated groups. Furthermore, the control and treatment groups' tissues were histopathologically examined to see if the two doses of RES-loaded poloxamer 407 and natural RES may produce tissue damage, inflammation, or lesions in the liver and kidneys (Figure 7). Examination revealed that mice treated with RES-loaded poloxamer 407 or with natural RES showed no visible histological abnormalities (Figure 7). As a result, this examination indicated that employing RES-loaded poloxamer 407 for in vivo administrations might be propitious. Previous studies are indicating the possibility of RES to protect the heart, kidney, lung, and spermatogenesis from the influence of oxidative side effects of radiation and the toxic effects of infrared rays. As previously mentioned it is a very effective antioxidant agent with impressive results and protects the body from wide types of cancer inducers as well as diabetes [43]. Previous studies have explained that RES can stimulate Sirt1 gene expression that enhances DNA repair ability against clastogenic agents [44]. Moreover, in another study Cyclosporine, an immunosuppressive drug, exhibits a toxic effect on renal and vascular systems. The RES therapy alleviates renal and vascular injury induced by cyclosporine when it was given for 7 days to the rats under experiment [45]. 

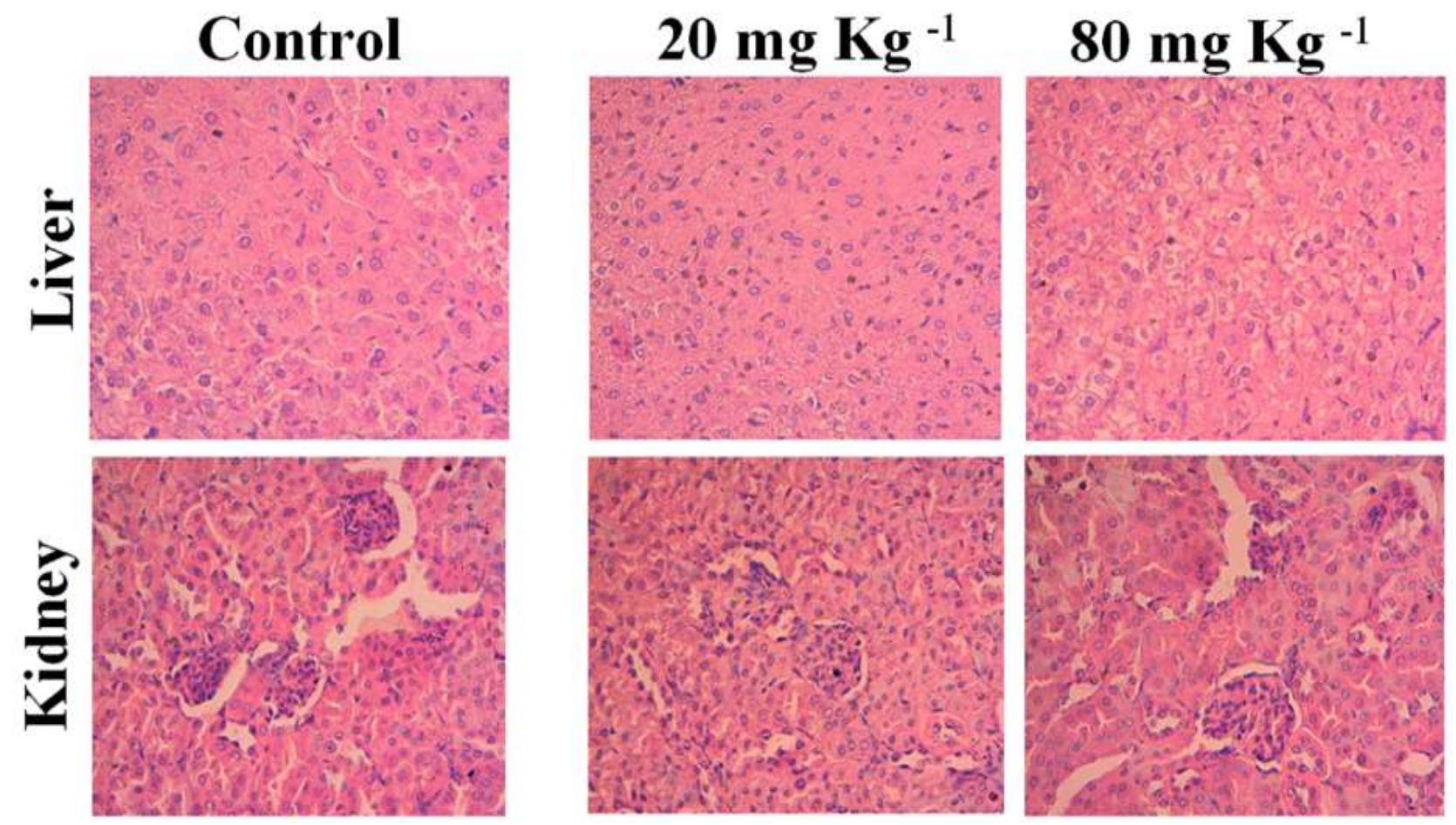

Figure 7: Histological images for liver and kidney of mice administrated the RES-loaded poloxamer 407 by intraperitoneal injection with two different doses (20, and $\left.80 \mathrm{mg} \mathrm{Kg}^{-1}\right)$. Magnification of images is 400x.

\section{Conclusions}

The results of this study show that the formulation of natural RES to RES in nano-structure (RES -loaded poloxamer 407) was effectively synthesized using the precipitation approach. Furthermore, certain trials were undertaken to demonstrate the bio-viability of the novel construct, and it was shown to be antioxidant effective, safe, and biocompatible for human red blood cells, with no toxicity and no harmful side effects or behavioral abnormalities in the administrated mice. It was also put to the test in terms of retaining the DNA molecule, with promising results.

\section{Conflict of Interest}

The authors declare that there are no conflicts of interest regarding the publication of this manuscript.

\section{References}

[1] American Cancer Society. Global Cancer Facts \& Figures 3rd Edition. American Cancer Society, vol. 800, pp. 1- 64, 2015.

[2] G. M. Cragg, and D. J. Newman, "Natural products: a continuing source of novel drug leads," Biochimica et Biophysica Acta, vol. 1830, pp. 3670-3695, 2013.

[3] M. J. R. Howes, and M. S. Simmonds, "The role of phytochemicals as micronutrients in health and disease," Current Opinion in Clinical Nutrition and Metabolic Care, vol. 17, pp. 558-566, 2014.

[4] N. Pujara, K. Y. Wong, Z. Qu, et al., "Oral delivery of $\beta$-lactoglobulin-nanosphere-encapsulated resveratrol alleviates inflammation in winnie mice with spontaneous ulcerative colitis," Molecular Pharmaceutics, vol. 18, pp. 627-640, 2021.

[5] L. Wang, H. Zhao, L. Wang, et al., "Effects of selected resveratrol analogues on activation and polarization of lipopolysaccharide-stimulated BV-2 microglial cells," Journal of Agricultural and Food Chemistry, vol. 68, pp. 3750-3757, 2020.

[6] S. Liu, Y. Li, F. Yi, et al., "Resveratrol oligomers from Paeonia suffruticosa protect mice against cognitive dysfunction by regulating cholinergic, antioxidant and anti-inflammatory pathways," Journal of Ethnopharmacology, vol.260, p.112983, 2020. 
[7] Y. Abba, H. Hassim, H. Hamzah, et al., "Antiviral activity of resveratrol against human and animal viruses," Advances in virology, vol. 2015, pp.1-7, 2015.

[8] D. Vervandier-Fasseur, and N. Latruffe, "The potential use of resveratrol for cancer prevention," Molecules, vol. 24, p. 4506, 2019.

[9] Y. Han, H. Jo, J. H. Cho, et al., "Resveratrol as a tumor-suppressive nutraceutical modulating tumor microenvironment and malignant behaviors of cancer," International journal of molecular sciences, vol. 20, p. 925, 2019.

[10] M. Jang, L. Cai, G. O. Udeani, et al., "Cancer chemopreventive activity of resveratrol, a natural product derived from grapes," Science, vol. 275, pp. 218-220, 1997.

[11] G. J. Soleas, L. Grass, P. D. Josephy, et al., "A comparison of the anticarcinogenic properties of four red wine polyphenols," Clinical biochemistry, vol. 35, pp.119-124, 2002.

[12] G. J. Soleas, J. Yan, and D. M. Goldberg, "Ultrasensitive assay for three polyphenols (catechin, quercetin and resveratrol) and their conjugates in biological fluids utilizing gas chromatography with mass selective detection," Journal of Chromatography B: Biomedical Sciences and Applications, vol. 757, pp. 161-172, Y..' .

[13] J. A. Giménez-Bastida, M. Á. Ávila-Gálvez, J. C. Espín, et al., "Conjugated Physiological Resveratrol Metabolites Induce Senescence in Breast Cancer Cells: Role of p53/p21 and p16/Rb Pathways, and ABC Transporters," Molecular nutrition \& food research, vol. 63, p. 1900629, 2019.

[14] S. K. Singh, S. Banerjee, E. P. Acosta, et al., "Resveratrol induces cell cycle arrest and apoptosis with docetaxel in prostate cancer cells via a p53/p21WAF1/CIP1 and p27KIP1 pathway," Oncotarget, vol. 8, p. 17216, 2017.

[15] A. O. Elzoghby, S. A. El-Lakany, M. W. Helmy, et al., "Shell-cross linked zein nanocapsules for oral codelivery of exemestane and resveratrol in breast cancer therapy," Nanomedicine, vol. 12, pp. 2785-2805, 2017.

[16] Z. Song, Y. Shi, Q. Han, et al., "Endothelial growth factor receptor-targeted and reactive oxygen speciesresponsive lung cancer therapy by docetaxel and resveratrol encapsulated lipid-polymer hybrid nanoparticles," Biomedicine and Pharmacotherapy, vol.105, pp. 18-26, 2018.

[17] D. Rossi, A. Guerrini, R. Bruni, et al., "trans-Resveratrol in nutraceuticals: issues in retail quality and effectiveness," Molecules, vol. 17, pp. 12393-12405, 2012.

[18] N. Bertrand, J. Wu, X. Xu, et al., "Cancer nanotechnology: the impact of passive and active targeting in the era of modern cancer biology," Advanced drug delivery reviews, vol. 66, pp. 2-25, 2014.

[19] G. M. Sulaiman, M. S. Jabir, and A. H. Hameed, "Nanoscale modification of chrysin for improved of therapeutic efficiency and cytotoxicity," Artificial cells, nanomedicine, and biotechnology, vol. 46, (sup1), pp. 708-720, 2018.

[20] J. Hao, B. Guo, S. Yu, et al., "Encapsulation of the flavonoid quercetin with chitosan-coated nano-liposomes," LWT - Food Science and Technology, vol. 85, pp. 37-44, 2017.

[21] A. G. Georgakilas, A. A. Konsta, E. G. Sideris, et al., "Dielectric and UV spectrophotometric study of physiocochemical effect of ionizing radiation on mammalian macro molecular DNA," Transaction Dielectrics and Electrical Insulation, vol. 8, pp. 549-555, 2001.

[22] G. M. Sulaiman, H. M. Waheeb, M. S. Jabir, et al., "Hesperidin loaded on gold nanoparticles as a drug delivery system for a successful biocompatible, anti-cancer, anti-inflammatory and phagocytosis inducer model," Scientific reports, vol.10, pp. 9362, 2020.

[23] H. Chen, A. Dorrigan, S. Saad, et al., "In vivo study of spherical gold nanoparticles: inflammatory effects and distribution in mice," PloS one, vol. 8 pp. e58208, 2013.

[24] X. Zhu, X. Zeng, X. Zhang, et al., "The effects of quercetin-loaded PLGA-TPGS nanoparticles on ultraviolet B induced skin damages in vivo," Nanomedicine: Nanotechnology, Biology and Medicine, vol. 12, pp. 623632, 2016.

[25] K. T. Savjani, A. K. Gajjar, and J. K. Savjani, "Drug solubility: importance and enhancement techniques." ISRN Pharmaceutics, vol. 2012, pp. 195727, 2012.

[26] H. Kaur, D. Singh, and G. Kaur, "Enhanced dissolution and antioxidant activity of chrysin nanoparticles employing co-precipitation as a technique," Pharmaceut Nanotechnol, vol. 3, pp. 205- 218, 2015.

[27] DJ. Huang, BX. Ou, RL. Prior, "The chemistry behind antioxidant capacity assays," J Agric Food Chem, vol. 53, pp. 1841-1856, 2005.

[28] İ. Gülçin, "Antioxidant properties of resveratrol: A structure-activity insight," Innovative food science \& emerging technologies, vol. 11, pp. 210-218. 2010. 
[29] Y. Fan, X. Zeng, J. Yi, et al., "Fabrication of pea protein nanoparticles with calcium-induced cross-linking for the stabilization and delivery of antioxidative resveratrol," International journal of biological macromolecules, vol. 152, pp. 189-198, 2020.

[30] G. R. Reddy, A. B. Morais, and N. N. Gandhi, "2, 2-Diphenyl-1-Picrylhydrazyl free radical scavenging assay and bacterial toxicity of protein capped silver nanoparticles for antioxidant and antibacterial applications," Asian Journal of Chemistry, vol. 25, pp. 9249-9254, 2013.

[31] R. Kohen, and A. Nyska, "Invited review: Oxidation of biological systems: oxidative stress phenomena, antioxidants, redox reactions, and methods for their quantification," Toxicologic Pathology, vol. 30, pp. 620650, 2002.

[32] B. Lipinski, "Hydroxyl radical and its scavengers in health and disease," Oxidative Medicine and Cellular Longevity, vol. 2011, p. 9, 2011.

[33] B. Kaleci, and M. Koyuturk, "Efficacy of resveratrol in the wound healing process by reducing oxidative stress and promoting fibroblast cell proliferation and migration," Dermatologic Therapy, vol. 33, pp. e14357, 2020.

[34] P. E. B. Ferreira, E. J. Beraldi, S. C. Borges, et al., "Resveratrol promotes neuroprotection and attenuates oxidative and nitrosative stress in the small intestine in diabetic rats," Biomedicine \& Pharmacotherapy, vol. 105, pp. 724-733, 2018.

[35] M. D. Scott, J. J. Van den Berg, T. Repka, et al., "Effect of excess alpha-hemoglobin chains on cellular and membrane oxidation in model beta-thalassemic erythrocytes," The Journal of Clinical Investigation, vol. 91, pp. 1706-1712, 1993.

[36] D. C. Castro, G. Gatti, S. E. Martín, et al., "Promising tamoxifen-loaded biocompatible hybrid magnetic nanoplatforms against breast cancer cells: synthesis, characterization and biological evaluation," New Journal of Chemistry, vol. 45, pp. 4032-4045, 2021.

[37] R. Katekar, G. Thombre, M. Riyazuddin, et al., "Pharmacokinetics and brain targeting of trans-resveratrol loaded mixed micelles in rats following intravenous administration," Pharmaceutical development and technology, vol. 25, pp. 300-307, 2020.

[38] M. Ferrali, C. Signorini, B. Caciotti, "Protection against oxidative damage of erythrocyte membrane by the flavonoid quercetin and its relation to iron chelating activity," FEBS Letters, vol. 416, pp. 123-129, 1997.

[39] Z. Huyut, M. R. Şekeroğlu, R. Balahoroğlu, "Characteristics of resveratrol and serotonin on antioxidant capacity and susceptibility to oxidation of red blood cells in stored human blood in a time-dependent manner," Journal of international medical research, vol. 46, pp. 272-283, 2018.

[40] J. Choi, V. Reipa, V. M. Hitchins, "Physicochemical characterization and in vitro hemolysis evaluation of silver nanoparticles," Toxicological Sciences, vol. 123, pp. 133-143, 2011.

[41] G. L. HW, M. Wortis, and R. Mukhopadhyay, "Stomatocyte-discocyte-echinocyte sequence of the human red blood cell: Evidence for the bilayer-couple hypothesis from membrane mechanics," Proceedings of the National Academy of Sciences, vol. 99, pp. 16766-16769, 2002.

[42] M. Suwalsky, F. Villena, and M. J. Gallardo, "In vitro protective effects of resveratrol against oxidative damage in human erythrocytes," Biochimica et Biophysica Acta (BBA)-Biomembranes, vol. 1848, pp. 76-82, 2015.

[43] M. Najafi, M. Cheki, P. Amini, "Evaluating the protective effect of resveratrol, Q10, and alpha-lipoic acid on radiation-induced mice spermatogenesis injury: A histopathological study, "International Journal of Reproductive BioMedicine, vol. 17, pp. 907, 2019.

[44] M. Currò, A. Trovato-Salinaro, A. Gugliandolo, "Resveratrol protects against homocysteine-induced cell damage via cell stress response in neuroblastoma cells," Journal of Neuroscience Research, vol. 93, pp. 149156,2015

[45] S. Bekpinar, E. Karaca, S. Yamakoğlu, "Resveratrol ameliorates the cyclosporine-induced vascular and renal impairments: possible impact of the modulation of renin-angiotensin system," Canadian journal of physiology and pharmacology, vol. 97, pp. 1115-1123, 2019. 